\title{
GERIATRIC EDITORIAL Obesity in the elderly: an emerging health issue
}

\author{
International Journal of Obesity (2012) 36, 1151-1152; doi:10.1038/ \\ ijo.2012.120
}

Epidemic obesity has been observed in the last few years in the USA, ${ }^{1}$ as well as in Europe. ${ }^{2}$ Epidemic obesity is not only a problem in childhood and adulthood but it also extends into older ages. The prevalence of obesity in older people has dramatically increased in recent years: in the United States, more than $30 \%$ of men and women aged 60 years and over are obese. A significant increase in the prevalence of extreme degrees of obesity also has been observed in older ages. ${ }^{1}$

The increased prevalence of obesity in older ages concurs with age-related changes in body composition, that is, a progressive increase in fat mass and a decline in lean mass and bone. There is also body fat redistribution, with an increase in visceral abdominal fat and a decrease in subcutaneous abdominal fat. ${ }^{3}$ Ectopic fat deposition within non-adipose tissue such as the skeletal and cardiac muscle, liver and pancreas also occurs with aging. ${ }^{3}$ Agerelated fat mass increase may occur without significant changes in body mass index (BMI) or body weight, but it is amplified by weight gain.

Although obesity, mainly visceral obesity, in adult ages has been shown to be clearly related to diabetes, hypertension, cardiovascular diseases and mortality, doubt still exists about its harm in the elderly. Studies on the relation between obesity in older ages and mortality show conflicting results, where a correlation between increased BMI and increased mortality in the elderly has been demonstrated in some but not all studies. Interpretation of these data is complicated by variation across studies, outcome considered, confounders accounted for in the analysis (especially control for smoking and concurrent illness), measure of obesity considered and length of study follow-up. ${ }^{3}$ Trajectory of weight from middle to old ages, as well as changes in weight in older ages, besides actual body weight, seems to be a strong predictor of mortality in old ages.

Clinicians taking care of older patients know that unintentional weight loss is independently related to higher mortality in the elderly. However, unintentional weight loss may happen in obese old people in several clinical conditions associated with obesity, such as diabetes, stroke and others, and it may be another confounder when evaluating the association between obesity in old age and mortality.

A consensus panel of the American Society for the Nutrition and the Obesity Society, after reviewing papers published between 1996 and 2005, stated that both overweight and obesity in the elderly are linked to physical disability. ${ }^{4}$ Overweight and obesity are also associated with higher risk of several chronic conditions such as diabetes, hypertension, stroke, heart disease and metabolic syndrome in the elderly. ${ }^{4}$

Despite all these adverse health issues associated with obesity, the issue of obesity in the elderly is frequently considered negligible by clinicians.

Actually, a consensus is still lacking even about the definition of obesity in the elderly. Doubt has been raised about the fact that recommendations for body weight and BMI do not change across ages. High values of waist circumference alone, or together with BMI, may be a better definition of obesity in the elderly. However, waist circumference cutoff points still need to be more thoroughly validated as predictors of morbidity and mortality in older ages.

Given the current increased prevalence of obesity in older aged persons in developed countries, the issue of obesity in the elderly should require more attention by researchers and physicians.

In this issue of the journal, papers evaluating different aspects of obesity in old ages are included. They cover many of the main topics of obesity in the elderly.

Midlife body weight has been the focus of two different papers, which evaluated, respectively, its association with disability ${ }^{5}$ and frailty. ${ }^{6}$ In a large study sample of subjects, a significant relation between BMI assessed at midlife and disability in old age was observed even after taking into account several covariates. ${ }^{5}$ After a 26-year follow-up, overweight and obese midlife men showed, respectively, 2.06 and 5.41 higher risk to become frail in old ages. Weight gain from midlife to old age was higher in subjects who became prefrail or frail in older ages. ${ }^{6}$

Behaviors responsible for weight gain in old ages have been evaluated in two large study samples. ${ }^{7,8}$ In a prospective study of post menopausal women with a 7-year follow-up, those who reported a moderate alcohol intake showed lower weight gain and had less risk of becoming overweight or obese. ${ }^{7}$ In a cohort of men aged 65 years at baseline, followed from 1988 to 1998, physical activity was protective against weight gain: ${ }^{8}$ subjects enrolled in $70 \mathrm{~min} /$ day of moderate intensity physical exercise were able to keep their weight stable over the 10-year follow-up period.

The hypothesis that the length of telomeres, DNA protein complexes at the end of chromosomes, is associated with obesity in old ages has been tested in the cohort of subjects enrolled in the Health $A B C$ study. ${ }^{9}$ Whether changes in telomere length are a cause or an effect of weight gain and obesity remains still a controversy.

De Caria et al. ${ }^{10}$ expand previous reviews of obesity in older ages by addressing the majority of the topics discussed above and adding subtopics such as treatment strategies and costs of obesity and interventions. The authors suggest that treatment strategies for obese older subjects should focus more on maintaining body weight and improving physical function, but avoiding weight loss. This conclusion, although reasonable, should take into account the statements of the consensus panel of the American Society for the Nutrition and the Obesity Society ${ }^{4}$ and the recent data published by Villareal et al. ${ }^{11}$ supporting the positive effect of moderate weight loss in the elderly. Caution concerning weight loss in the elderly is supported by the so called Obesity Paradox, where obesity in older people is beneficial or neutral rather than harmful. It is indubitable that higher adiposity may confirm higher protection against catabolic stress by supplying nutritional reserves. Several mechanisms may be responsible for the Obesity Paradox. ${ }^{3}$ Hong et al. ${ }^{12}$ added complexity to the topic of the Obesity Paradox showing that fat mass, as evaluated by dual X-ray absorptiometry, was strongly related to higher mortality in subjects without organic pollutants, whereas the relationship was opposite in the presence of organic pollutants with the latter results supporting, and the former denying the Obesity Paradox.

Identification of different phenotypes of obesity in the elderly (for example, sarcopenic obese subjects), as well as greater focus on the health-specific status of each old obese subject, should be the basis of a rational treatment of obesity in the elderly. In some cases (for example, old patients affected by diabetes, 
hypertension, knee pain), carefully supervised moderate weight loss with modest energy restriction, coupled with physical exercise (both aerobic and resistance), may be beneficial.

Prevention of obesity, and in particular of weight gain in adults from middle age to old age, is very important. Population lifestyle intervention targeting the increase of physical activity and the improvement of dietary habits appears to be the most effective treatment strategy in helping to decrease or maintain body weight and improve function and fitness in the elderly people. ${ }^{10}$

\section{CONFLICT OF INTEREST}

The authors declare no conflict of interest.

M Zamboni and G Mazzali Geriatric Division, Department of Medicine, University of Verona, Verona, Italy E-mail: mauro.zamboni@univr.it

\section{REFERENCES}

1 Flegal MK, Carroll MD, Kit BK, Ogden CL. Prevalence of obesity and trends in the distribution of body mass index among US adults, 1999-2010. JAMA 2012; 307 491-497.

2 Micciolo R, Di Francesco V, Fantin F, Canal L, Harris TB, Bosello O et al. Prevalence of overweight and obesity in Italy (2001-2008): is there a rising obesity epidemic? Ann Epidemiol 2010; 20: 258-264.
3 Zamboni M, Mazzali G, Zoico E, Harris TB, Meigs JB, Di Francesco V et al. Health consequencs of obesity in the elderly: a review of four unresolved questions. Int $J$ Obes 2005; 29: 1011-1029.

4 Villareal DT, Apovian CM, Kushner RF, Klein S. American Society for Nutrition and NAASO, The Obesity Society. Obesity in older adults: technical review and position statement of the American Society for Nutrition and NAASO, The Obesity Society. Am J Clin Nutr 2005; 82: 923-934.

5 Backholer K, Pasupathi K, Wong E, Hodge A, Stevenson C, Peeters A. The relationship between body mass index prior to old age and disability in old age. Int Obesity 2012; 36: 1180-1186.

6 Strandberg TE, Sirola J, Pitkala KH, Tilvis RS, Strandberg AY, Stenholm S. Association of midlife obesity and cardiovascular risk with old age frailty. A 26-year follow-up in initially healthy men. Int J Obesity 2012; 36: 1153-1157.

7 Thomson CA, Wertheim BC, Hingle M, Wang L, Neuhouser ML, Gong Z et al Alcohol consumption and body weight change in postmenopausal women. Int $J$ Obesity 2012; 36: 1158-1164.

8 Shiroma EJ, Sesso HD, Lee I-M. Physical activity and weight gain prevention in older men. Int J Obesity 2012; 36: 1165-1169.

9 Njajou OT, Cawthon RM, Blacburn EH, Harris TB, Sanders JL, Newman AB et al. for the Health $A B C$ study. Shorter telomeres are associated with obesity and weight gain in the elderly. Int J Obesity 2011; 18: 1-4.

10 DeCaria JE, Sharp C, Petrella RJ. Scoping review report: obesity in older adults. Int J Obesity 2012; 36: 1141-1150.

11 Villareal DT, Chode S, Parimi N, Sinacore DR, Hilton T, Armamento-Villareal R et al Weight loss, exercise, or both and physical function in obese older adults. N Engl J Med 2011; 364: 1218-1229.

12 Hong N-S, Kim K-S, Lee I-K, Lind PM, Lind L, Jacobs DR et al. The association between obesity and mortality in the elderly differs by serum concentrations of persistent organic pollutants: a possible explanation for the obesity paradox. Int $\mathrm{J}$ Obesity 2012; 36: 1170-1175. 